$\underline{\text { Review Article }}$

\title{
A REVIEW OF LABORATORY DIAGNOSIS AND TREATMENT OF LEPTOSPIROSIS
}

\author{
WALEED AL-ORRY1,2, MOUSTAPHA ARAHOU ${ }^{1}$, RACHIDA HASSIKOU ${ }^{1}$, ZAKARIA MENNANE² \\ ${ }^{1}$ Laboratory of Botany, Mycology and Environment, Department of Biology, Faculty of Sciences, Rabat, Morocco, ${ }^{2}$ Department of Medical \\ Bacteriology, National Institute of Hygiene, Ibn Battuta Avenue, B. P. 769, Agdal, Rabat 11000, Morocco \\ Email: binahmed1977@gmail.com
}

Received: 13 Aug 2016 Revised and Accepted: 05 0ct 2016

\begin{abstract}
Leptospirosis is an infectious disease affect human and animal. The diagnosis is challenging due to none specific clinical features, lack of awareness and poor access to health care. Therefore the disease becomes underreported. Early diagnosis is essential because antibiotic treatment is most effective when it is initiated early. However, laboratories tests for early diagnosis still lacking. The disease may be demonstrated by direct or indirect methods. Direct methods are the detection of the organism or its components and indirect methods are the detection of specific antibodies of the organism. This review highlights the major diagnostic techniques in leptospirosis with their advantages and disadvantages as well as the treatment. The search criteria focused on the thesis we obtained and searching in the websites (Google Scholar, PubMed, Science Direct and LibGen, etc.) using the keywords: leptospirosis, leptospira, zoonotic diseases, PCR, Elisa, MAT, SAT, Darkfield microscopic, leptospirosis diagnostic, leptospira treatment, Doxycycline and tropical diseases.
\end{abstract}

Keywords: Leptospirosis, Diagnosis, Treatment, Zoonoses, Tropical diseases, MAT

(C) 2016 The Authors. Published by Innovare Academic Sciences Pvt Ltd. This is an open access article under the CC BY license (http://creativecommons.org/licenses/by/4. 0/] DOI: http://dx.doi.org/10.22159/ijpps.2016v8i12.14688

\section{INTRODUCTION}

Leptospirosis is a zoonotic disease of increasing prevalence, worldwide distribution, and with potentially serious consequences for human and animal health. Transmission occurs from exposure to urine or aborted tissues of infected animals either directly or via contact with contaminated water or soil $[1,2]$. Leptospirosis is generally underreported due to nonspecific symptoms, lack of awareness, challenging diagnostics and poor access to health care [3]

As a result of the variety of clinical and often "flu-like" symptoms, human leptospirosis is often undiagnosed or misdiagnosed as other illnesses with febrile syndromes (e. g. aseptic meningitis, influenza, hepatic disease and Hantavirus infections) $[4,1]$. This is also the case in domestic animals where most cases are difficult to diagnose clinically due to a non-specific clinical presentation or unapparent clinical signs with host-adapted serovars. Therefore, diagnosis of leptospirosis in both humans and animals cannot be made with confidence without laboratory confirmation [4].

Early diagnosis is essential because antibiotic treatment is most effective when it is initiated early in the course of the disease. However, many experts agree that adequate laboratory tests for early diagnosis are still lacking [5]. It is not necessary to confirm the diagnosis or wait for the result of the tests before starting treatment. The clinical assessment and epidemiologic history are more important. Early recognition and treatment is more important to prevent complications of the severe disease and mortality [6].

Leptospirosis of man and animals is investigated by direct and indirect laboratory methods [7].

1. Direct methods are detection of the organism or its components in body fluid or tissues by isolation of leptospires in cultures, identification of Leptospira species antigens in tissue and body fluids using such methods like immunofluorescence staining immunochemistry, immune-peroxidase staining, silver staining and various methods of Polymerase Chain Reaction (PCR) [4, 8]. Direct visualization of leptospirae in blood or urine by dark field microscopic examination has been used for direct diagnosis [7]

2. Detection of specific antibodies to leptospires (serological diagnosis) is indirect evidence [9]. These methods are either methods detecting serum antibodies without discriminating on serovars, such as various ELISA tests, indirect immunofluorescence, the spot agglutination test or methods reliably identifying the infecting serovars such as the Microscopic Agglutination Test (MAT) [10].

The collection of appropriate specimens and selection of tests for diagnosis depend on upon the timing of collection and the duration of symptoms [1]. In humans, the first stage of the biphasic illness occurs before antibodies develop. Leptospira may be found in the blood, cerebrospinal fluid, urine or tissue samples. Early cases must be diagnosed with assays that detect the organism, its antigens or nucleic acids. However, in many cases leptospirosis is diagnosed by serology especially the MAT or ELISAs [11]. Diagnostic testing should be requested for patients in whom there is a high index of suspicion for leptospirosis, based either on signs and symptoms or on occupational, recreational or vocational exposure to animals or environments contaminated with animal urine [12]. Early diagnosis of leptospirosis is critical because of the risk of severe complications of the disease including pancreatitis, lung and intracranial hemorrhages, which requires intense care therapy [13]

\section{What kind of sample, what test and when to test?}

The choice for the use of a diagnostic test will depend on a number of factors including its diagnostic accuracy, financial feasibility technical or practical feasibility and the need for an early and/or rapid result [14]. Infection by pathogenic leptospires may be divided into two stages (fig. 1). The first stage of leptospirosis is leptospiraemia or the acute stage, which lasts from 3 to $10 \mathrm{~d}$ with headaches and myalgia. During that stage, leptospires are found in blood in a decreasing number until $15 \mathrm{~d}$ after onset of symptoms. Samples must be collected up to $2 \mathrm{~d}$ after initiating anti-biotherapy to detect leptospires [15]. The chances of recovery of leptospira from blood or other tissues or body fluids is usually high during this stage [9]. The absence of detection of leptospiral antigen or DNA in confirmed cases of leptospirosis can be attributed to a low or a short leptospiremia during the acute phase of the disease, to blood samples taken late in the disease or to the administration of antibiotics [14].

The second stage (convalescent) or immune stage called leptospiruria usually occurs during the second week after onset of symptoms [15]. This stage is characterized by excretion of leptospires in the urine and appearance of antibodies in the serum. Leptospires are cleared from the bloodstream as and the titers of IgM class antibodies increase [16]. Antibodies generally reach 
maximum levels within two to three weeks then gradually recede [1] But may remain detectable for two to ten years in humans and for similar periods or for a lifetime at low levels in animals (particularly in reservoir hosts) [4].

Generally, until the 6th day, only PCR from blood samples can be performed. From 6th to 10th day it is possible to perform ELISA if PCR is not available. From the 11th day, the PCR should be performed on samples of CSF or urine and it is possible to perform MAT or ELISA [17].

The definitive diagnostic test is the recovery of leptospires from clinical specimens either by a culture which is insensitive and slow, by immune-histochemical staining or by showing the presence of leptospiral DNA by PCR [18].

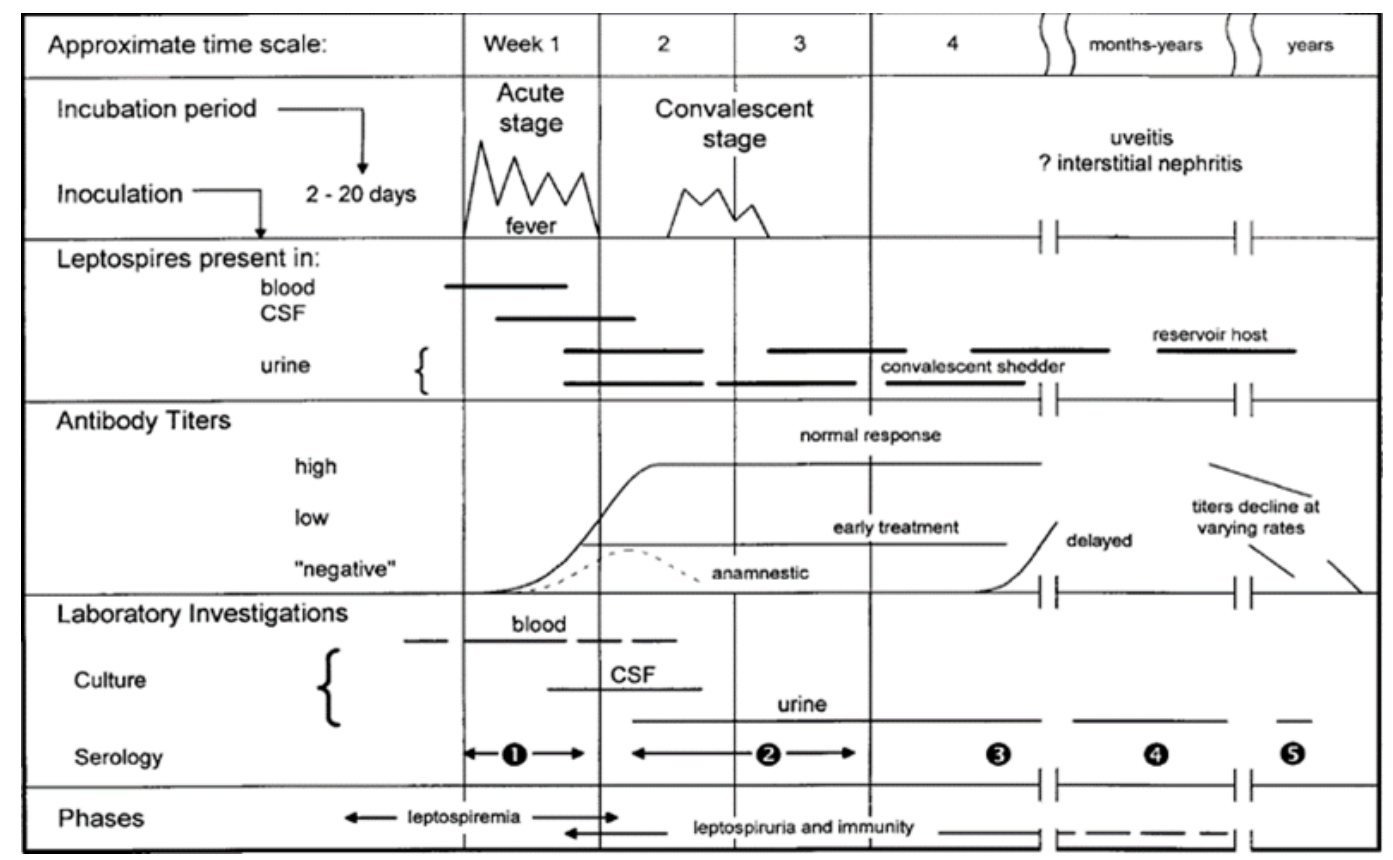

Fig. 1: Schematic representation of the biphasic nature of leptospirosis and relevant diagnostic investigations at different stages of disease. For serology specimens 1 and 2 are acute-phase specimens, 3 is a convalescent-phase specimen which may facilitate detection of a delayed immune response, 4 and 5 are follow-up specimens which can provide epidemiological information, such as the presumptive infecting serogroup. This fig. was from [1]

\section{Direct examination for leptospires}

\section{Darkfield microscopic examination}

Leptospires may be demonstrated by direct microscopic observation of clinical specimens [5]. Darkfield microscopic examination (DFM) of body fluids such as blood, urine, CSF and dialysate fluid can be used to rapidly detect the presence of leptospires as well as from tissues removed for surgical or experimental reasons from animals or necropsy specimens, tissues from carcasses or abortion products [1,19]. This technique has advantages for early diagnosis [5]. Although it is useful in situations where laboratory resources are limited, DFM has low sensitivity and specificity and provides no information on the infecting serovar [16].

The bacterial load in blood ranges from $10^{2}$ to $10^{6}$ Leptospira per milliliter in the acute phase. In theory, leptospirosis can, therefore, be diagnosed by dark-field microscopy of blood taken during the first week of illness [14]. Approximately $10^{4}$ leptospires $/ \mathrm{ml}$ is the detection threshold for one cell per field to be visible [5]. The organism is only present in blood during a short period during the acute stage of the disease; the concentration is too low to allow detection by direct microscopy and shedding in urine is intermittent [20]. The risk of false positives due to misinterpretation of fibrin or protein threads, cell debris and other artefacts can be high even for experts. Thus, results of direct examination may be supported by another laboratory irrespective of positive results. The result is also affected by the timing of sample collection and the skill of the laboratory personnel [5].

To increase the sensitivity of direct microscopic examination of leptospires in veterinary specimens, a variety of staining methods have been used including immunofluorescence staining $[4,5]$. Finally, although it is relatively inexpensive, DFM test requires a dark-field microscope which is rarely available or affordable in resource-limited settings [14].

\section{Culture}

Requires special media, leptospires can be isolated from whole blood (within $7 \mathrm{~d}$ of onset), cerebrospinal fluid (CSF) during the acute illness (4-10 d from onset) and from urine (after the 7th day and only if inoculated into special media within $2 \mathrm{~h}$ of voiding). Clinical or autopsy specimens (e. g., punch biopsy of the kidney) should be submitted fresh or frozen [12]. Isolation of leptospires from clinical specimens is the strongest evidence for confirmatory diagnosis and definite proof of infection. Circulating serovars can be identified, local isolates can be used as antigens in MAT and in vaccine development [9].

Samples for culture should be collected prior to the administration of antibiotics [21]. Samples should be stored and transported at ambient temperatures since low temperatures are detrimental to pathogenic leptospires [22]. Venous blood is collected by means of an aseptic technique and ideally inoculated at the bedside into blood culture bottles containing culture medium for Leptospira. Small inocula consisting of a few drops of blood are inoculated into several tubes each containing $5 \mathrm{ml}$ of a suitable medium. Large inocula will inhibit the growth of leptospires. Cultures should be incubated at 30 ${ }^{\circ} \mathrm{C}$ and checked regularly for a period of 4-6 mo [23]. Incubation for up to $13 \mathrm{w}$ at $30{ }^{\circ} \mathrm{C}$ with a weekly examination by dark field microscopy (DFM) is necessary before cultures can be discarded as negative [24].

The growth of leptospires is often slow on primary isolation, but pure subcultures in liquid media usually grow within 10 to $14 \mathrm{~d}$ [1]. Subculture should be made within $48 \mathrm{~h}$ to minimize the inhibitory effect of the selective agents on leptospires [9]. 
A commonly used medium for culture is Ellinghausen-McCulloughJohnson-Harris (EMJH) medium which contains 1\% bovine serum albumin and polysorbate 80 (source of long-chain fatty acids). This culture is expensive and technically demanding. Thus the medium is stocked in few laboratories [4]. Several liquid media containing rabbit serum were described [1]. Agar may be added at low concentrations $(0.1$ to $0.2 \%)$ and growth on media solidified with agar has been reported. This medium is available commercially from several manufacturers [1].

For urine samples, fresh midstream urine is collected and inoculated immediately [23]. The highest success rate of culturing from urine is 14-28 d after infection when significant leptospiruria is seen. Optimum growth $\mathrm{pH}$ for leptospires is 6.8-7.4, the organisms die or lyse rapidly in acid urine [4]. Therefore, voided human urine and acidic animal urine should be processed immediately by centrifugation and the sediment resuspended with phosphatebuffered saline afterward to neutralize the $\mathrm{pH}[4,1]$. The culture of leptospires from urine may be poorly sensitive due to the intermittent shedding of bacteria in both human and animal cases, problems with contamination and the acidic urine environment [25].

Leptospira spp. stain poorly with the Gram stain and are not observed by microscopy unless special stains or methods are employed. Silver staining or immunogold-silver staining is sometimes useful as an adjunct technique. Dark field microscopy can also be used to detect Leptospira [26].

Although culture provides valuable information and material, it is only positive in a minority of cases. Culture is rarely performed in routine clinical practice since this may take several months and requires considerable expertise, which places it within the domain of specialist reference centers [27]. For this reason, culture is not considered useful as a routine test for diagnosis of individual patients but remains important for epidemiological purposes and definitive [26, 24].

\section{Polymerase chain reaction (PCR)}

Isolation of leptospires from clinical specimens requires a couple to several weeks for growth and current serological tests exhibit low sensitivity in the acute phase which limits their contribution to early diagnosis, require paired sera for definitive serodiagnosis and recent findings suggest low specificity of these tests in some highly [5]. To overcome all these limitations polymerase chain reaction (PCR) have been used, which is considered sensitive and specific for the rapid detection of Leptospira in clinical samples. Evaluation of PCR-based studies for the identification and diagnosis of leptospirosis has been reported elsewhere, in humans and animals [13, 28, 29, 30]. It has been developed for the rapid detection of Leptospira DNA and has the potential to make a dramatic impact in diagnosing leptospirosis [25].

PCR detects DNA in blood in the first 5-10 d after the onset of the disease and up to the 15th day and allows detection of leptospires in culture negative blood if the patient has received an effective antimicrobial drug but have not cleared nonviable organism [21]. Leptospiral DNA has been amplified from serum, urine, aqueous humor, CSF, water and a number of organs post mortem. [31, 32]. Depending on several factors, such as primer sets and disease stage at the time of analysis [33].

The major advantage of PCR is the prospect of confirming the diagnosis during the early acute (leptospiremia) stage of the illness, before the appearance of immunoglobulin M (IgM) antibodies when treatment is likely to have the greatest benefit. In fulminating cases in which death occurs before seroconversion, PCR may be of great diagnostic value [7]. In this regard, PCR-based diagnosis of leptospirosis should be made available for clinicians for the early diagnosis and prompt treatment of the disease [13]. It has been demonstrated that both conventional and real-time PCR are useful for early diagnosis during which antibody production has not begun [5]. However, PCR was recommended to be used in combination with serological tests; this improves the sensitivity of the diagnosis of leptospirosis in the first phase of the disease [33].

PCR assay can differentiate between pathogenic and non-pathogenic species [18]. More sensitive and have a higher specificity than conventional methods such as culture and dark-field microscopy
[32]. However, the sensitivity of PCRs for DNA detection in blood declines over the course of the disease as a corollary [5]. Numerous studies document its high specificity and sensitivity with the capability of detecting as few as 10 organisms in a sample. [25]. However, Leptospires antigens or DNA may not be detected in blood in some cases of leptospirosis maybe because of a weak or short leptospiremia during the acute stage or because of a late sampling or because of antibiotic administration which rapidly eliminates leptospires from blood [15].

PCR on urine samples can differentiate the carrier or shedding state while serological tests can only detect antibody regardless of infection status at the time. A limitation of PCR-based diagnosis of leptospirosis is the current inability of PCR assays to identify the infecting serovar, while this is not significant for individual patient management [34]. Real-time PCR, either using SYBR Green or Taqman technology has the advantage that it gives a result much faster than conventional PCR and is less prone to contamination [14].

\section{Serological diagnosis (Indirect methods)}

Most cases of leptospirosis are diagnosed serologically [35]. Using serum or plasma for the serological tests gives equivalent results, but serum should be used preferentially [15]. Antibodies are detectable in the blood approximately 5 to $7 \mathrm{~d}$ after the onset of symptoms [1]. Reactions persist for months or years. Therefore, persistent antibodies allow retrospective diagnosis [36]. In that moment, due to the lower probability of leptospires detection in blood, serologic methods become required [37].

The antibodies usually develop within 2-12 d after the onset of illness. IgM antibody starts appearing early in the course of the disease and reaches detectable levels within one week or as early as on the third or fourth day of illness. They reach peak levels during the third or fourth week and then decline slowly over months and become undetectable within six months (fig. 2). However, rarely IgM may persist at a low level for several years [9]. IgG antibodies appear later than IgM and reach peak level after few weeks of illness and may persist at a low level for years [9].

Although, Enzyme-linked immunosorbent assay (ELISA) and MAT are the most commonly available serological tests, the definitive serological investigation in leptospirosis remains the microscopic agglutination test (MAT) $[38,1]$.

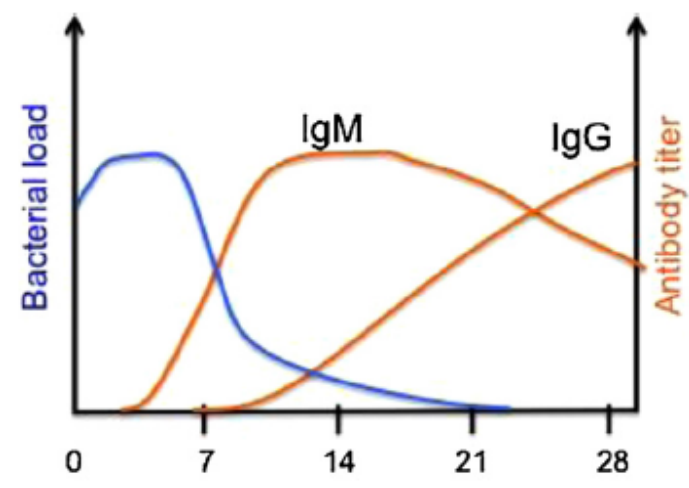

Number of days after onset of symptoms

Fig. 2: IgM and IgG, fig. from [15]

\section{Microscopic agglutination test (MAT)}

The microscopic agglutination test (MAT) or Martin and Pettit test was developed almost one century ago at the Pasteur Institute after the first isolation of Leptospira. It remains the gold standard for the serological diagnosis of leptospirosis and generally provides an indication of the presumptive serovar or serogroup responsible for an infection $[15,35]$. The test remains the cornerstone of serodiagnosis of leptospirosis and a helpful tool in understanding the epidemiology of the disease [20]. It is the most appropriate test 
to employ in epidemiological serosurveys since it can be applied to sera from any animal species and the range of antigens used can be expanded or decreased as required [1]. It has been used as the test of choice in outbreaks and sporadic cases and has also been useful in retrospective studies in confirming leptospirosis cases and identifying the prevalent serovar during that period [39].

MAT detects both class $M$ and class $G$ antibodies and cannot differentiate between current recent or past infections [21]. It was reported to have a sensitivity of $41 \%$ during the 1 st week, $82 \%$ during the 2nd to 4 th week and $96 \%$ beyond the 4 th week of illness [21]. The method has a good specificity because the presence of heterologous antibodies is not interfering in the results [10]. However, its sensitivity is low compared to Elisa/SAT (Slide agglutination test) in the acute phase [40].

Although, approximately 7 to $10 \mathrm{~d}$ after the onset of symptoms, antibodies can be detected by the MAT [4]. It requires expertise, pathogen containment level 2 (PC2) laboratory safety and it is timeconsuming as live Leptospira culture is used as the antigen. [3]. In this test, serial dilutions of serum are incubated with cultures of specific Leptospira serovars (antigen). After incubation, the serum/antigen mixtures are examined by dark field microscopy for agglutination and the titers are determined. A serum is considered as positive at a given dilution and for the tested antigen if at least $50 \%$ of leptospires are agglutinated compared to a control antigen without serum $[15,1]$.

The range of antigens used should include serovars representative of all serogroups moreover all locally common serovars and panels of live leptospires belonging to different serovars must be maintained in the laboratory $[1,21]$. At the Leptospirosis National Reference Center (French acronym CNRL) (Pasteur Institute) 24 strains are used including non-pathogenic strain Leptospira biflexa strain Patoc1 which has for particularity to cross-react with several antigens of pathogenic serogroups. A smaller panel of antigens (according to French guidelines for biological procedures the test should be performed with a minimum of nine antigens) may lead to non-detection of serogroups not presents in the panel [15]. An incomplete panel should be responsible for false negative results [21].
MAT is a complex test to control, perform and interpret. Interpretation of the MAT is complicated by the high degree of crossreaction that occurs between different serogroups especially in acute-phase samples [34]. As well as the repeated weekly subculture of large numbers of strains presents hazards for laboratory workers and laboratory-acquired infections have been reported. Because the sensitivity of the MAT is low in the acute phase, the test is ideally performed on paired serum samples (acute and convalescent). However, in actual practice obtaining paired samples from patients is very difficult [1]. Furthermore, many laboratories and hospitals do not have the facilities required to perform the MAT [35]. Therefore, it use is restricted to a few reference laboratories and a number of rapid screening tests for antibody detection in acute infection have been developed $[5,41]$.

\section{Titers of the MAT}

The standard criterion for a positive MAT are a fourfold increase between paired sera in antibody titre (between acute and convalescent serum samples) or a conversion from seronegativity to a titre of $1 / 100$ or above [18]. A titer of at least 1: 800 in the presence of compatible symptoms is strong evidence of recent or current infection [41].

In endemic areas, a titer of $1 / 100$ or $1 / 200$ is considered low while high titer is usually $>1 / 400$ (some consider $1 / 800$ or $1 / 1600$ as diagnostic criteria). In non-endemic areas, $1 / 100$ titer is taken as diagnostic criteria [42]. The titre cut-off of $1: 48$ is recommended to determine exposure to leptospires but not for clinical disease [3].

\section{Interpretation of diagnostic MAT}

It is difficult to confirm acute infection from a single serum sample. A low titer equal to 100 in a febrile patient may indicate current infection in areas where leptospirosis is uncommon [5]. The modified Faine's criteria which include Elisa IgM and other rapid tests along with culture and MAT for diagnosis of leptospirosis is the most practical guideline for Indian institutions (table 1). If MAT is available as a single test, positive rapid tests plus high titers in MAT can confirm the diagnosis of current leptospirosis. A negative rapid test with positive MAT might suggest past infection [43].

Table 1: Interpretation of MAT with ELISA and SAT, table from [40]

\begin{tabular}{lll}
\hline Elisa/SAT & MAT & Interpretation \\
\hline+ & Single high titer & Current infection \\
+ & - & Current infection \\
- & Single high titer & Past infection \\
+ & Seroconversion/ & Current infection \\
\hline
\end{tabular}

In a nonendemic area, any level of antibodies, however, low may signify leptospirosis in the 1 st week of a clinically compatible illness. The titer will rise in a second specimen taken after 3 to $7 \mathrm{~d}$. If the titer remains below 100 even on repeated testing, it may be assumed that it was due to the previous leptospirosis and not to current illness. A titre of 400 to 800 or more or a 4 -fold rise in titer between 2 tests (between sera taken five to $10 \mathrm{~d}$ ), is diagnostic when combined with a clinical illness compatible with leptospirosis [36, 3].

In endemic areas, the diagnosis will be confirmed if the titre rises on retesting, but will be negated if it is unchanged, assuming that the infecting serovar was included among the antigens for the MAT [36]. A high titer of 400 or more in an asymptomatic patient are generally accepted, but titers as high as 1600 or more have been recommended [5].

\section{Enzyme-linked immunosorbent assay (Elisa)}

Because of the complexity of the MAT, rapid screening tests for leptospiral antibodies in acute infection have been developed. Conventional serological methods such as enzyme-linked immunosorbent assay (Elisa) are widely used for the diagnosis of leptospirosis. Several IgM Elisa are available on the market based on the detection of antibodies against a total extract of leptospires.
Usually, the saprophytic strain L. biflexa which shares several surface antigens with pathogenic strains and pathogenic species have also been used $[34,44]$. The specificity and the sensitivity of these Elisa are quite variable. Theses reported variations may be due to differences in the studied population (previous exposure to pathogenic or environmental leptospires) [15].

Elisa is easy, safe and can detect IgM and IgG [45]. It can be used in humans and animals $[46,47]$. Elisa can be performed with minimal training and typically provides results in $2-4 \mathrm{~h}$ [14]. It has been recommended for the rapid diagnosis of leptospirosis in endemic areas [48]. Using human sera collected during the acute phase of infection IgM ELISA was reported to be more sensitive than the MAT [49]. ELISA is usually positive from day 6-8 earlier than the MAT [21]. Allowing the diagnosis to be confirmed and treatment to be initiated while it is likely to be most effective [15].

ELISA-based testing will be less expensive than MAT as it does not require the maintenance of live organisms and can be performed in any routine laboratory and further, unlike MAT does not require trained personnel and can be quantitated and is not prone to interobserver and intra-observer errors as in MAT [39]. Therefore, Elisa has been used as an alternative test to MAT for screening for leptospiral infection in both human and animals. Another advantage 
of ELISA over MAT is the serological response of IgM and IgG can be detected separately [4].

A patient's serum may be positive $5 \mathrm{~d}$ after onset of symptoms but not usually before this period (in cases where antibiotic treatment has been initiated this period may be increased) [23]. This confirms that when the result of the IgM-ELISA for the first sample is negative but the clinical findings are suggestive, or the agglutination test is positive, the second sample for IgM-ELISA testing should be examined [50].

Although, Immunoglobulin M enzyme-linked immunosorbent assay (IgM ELISA) presents high sensitivity, its specificity with crude antigens of pathogenic and nonpathogenic leptospires is not always adequate for routine tests. It has been shown that specific antibodies are detectable in patients who have been free of active disease for long periods [33].

Specificity of Elisa is affected by the antigen used in the assay, by the presence of antibodies due to previous exposure (in endemic regions) and by the presence of other diseases [34]. A positive Elisa gives no indication on the infecting serovar/serogroup and is not sufficient to diagnose a case of leptospirosis, it must be confirmed by MAT, PCR or culture [15].

\section{Slide agglutination test (SAT)}

Several attempts have been made to simplify and reduce the time, skill and expense required for serological testing [51]. Ideal test should be safe, rapid and simple, stable and accessible even to little and distant laboratories. It should also present good sensitivity rates and be indicated to the diagnosis of the acute form of leptospirosis [51]. SAT test was developed in 1958 by Galton et al. for the diagnosis of leptospirosis [52]. Stoenner and Davis have modified the preparation of plate antigens for leptospirosis diagnostic and concluded that this antigen could be used in rapid tests, obtaining similar sensitivities with the MAT in human, porcine and bovine sera [53]. SAT is inexpensive, can be performed more quickly, more easily than ELISA and MAT and could be used by the less well-equipped laboratories. It seems to be a convenient test for the initial diagnosis of leptospirosis and presenting high sensitivity in the acute phase [54]. It is more sensitive than the MAT and Elisa for early antibody detection in the course of the disease [50]. The main disadvantages of this test are that it is not suitable for epidemiological studies, identification of strains, assessment of the probable infecting serogroup and confirmation of illness for public health surveillance [51]. However, SAT can be performed to provide physicians with preliminary and rapid results. It is very easy to perform and usually take less than 5 min to perform [50].

\section{Other serological tests}

Other serological tests have been developed: complement fixation, counter immune-electrophoresis, indirect fluorescent antibody, indirect hemagglutination (IHA), sensitized erythrocyte lysis, latex agglutination (LA), macroscopic slide agglutination, microcapsule agglutination, and Patoc slide agglutination [21]. Nevertheless, these tests even if some are marketed, are rarely used and lack specificity or sensitivity [15].

\section{Treatment and prophylaxis}

Antibiotic therapy should be started as soon as the diagnosis of leptospirosis is suspected regardless of the phase of the disease or duration of symptoms [6]. However, the majority of patients, especially in endemic areas, do not develop a clinical syndrome. Of those with symptoms, $90 \%$ present with a flu-like, self-remitting disease that can easily run undetected. Five to nine percent develop a moderate clinical syndrome requiring hospitalization and 1-5\% develop the severe form of leptospirosis, usually manifested as the icterohaemorrhagic Weil's syndrome and pulmonary hemorrhagic form, the latter usually being an early event [55]. If a clinical diagnosis is made, the patient should be treated with doxycycline or penicillin. Treatment of the severe form of the disease is best undertaken in hospital [56].

Antibiotic treatment is effective within 7 to $10 \mathrm{~d}$ after infection and should be given immediately on diagnosis or suspicion [57]. The patient should be observed for evidence of renal failure and treated if necessary with hemodialysis. Patients with Weil's disease having hemorrhagic manifestation may require whole blood or platelet transfusion. Patients with MOF require to be observed in intensive care unit [57].

Usefulness of antimicrobial treatment is controversial however, penicillin and doxycycline are widely used for the treatment of leptospirosis in humans. To be effective, treatment should be started early during the acute stage of illness [3]. The drug of choice is benzylpenicillin by injection in the doses of five million units per day for five days. Patients who are hypersensitive to penicillin can be given erythromycin $250 \mathrm{mg}$ four times daily for five days. Doxycycline $100 \mathrm{mg}$ twice daily for ten days is also recommended. Tetracycline's and Doxycycline are effective but contraindicated in patients with renal insufficiency, in children and pregnant or breastfeeding women $[58,59]$. Erythromycin has also been found effective in severe leptospirosis [57]. Injection of Hydrocortisone $100 \mathrm{mg}$ every 8 hourly is also given in severe cases [60]. Antibiotic therapy should be completed for $7 \mathrm{~d}$ except for azithromycin dihydrate which could be given for $3 \mathrm{~d}[6]$.

Doxycycline (hydrochloride and hyclate) has been used as a chemoprophylactic agent for short time exposure, but it cannot be recommended for continuous routine use or for a long-term occupational exposure [60]. The duration of prophylaxis depends on the degree of exposure and the presence of wounds. Individuals should continue to monitor themselves for fever and other flu-like symptoms and should continue to wear personal protective measures since antibiotic prophylaxis is not $100 \%$ effective. The decision to give prophylaxis depends on the risk exposure assessment [6]. K. S. Uma and others found the drug Seenthil sarkarai was shown in the lesser concentration of MIC compare to the drug Nilavembu kudineer for anti-leptospiral activity [61].

Finally, Doxycycline has been used as a chemoprophylactic agent for short-term exposure and mild disease, but it cannot be recommended for continuous routine use or for long-term occupational exposure. Ampicillin and amoxicillin are also recommended in mild disease, whereas penicillin $\mathrm{G}$ and ampicillin are indicated for severe disease(table 2) [9, 18]. Prompt antibiotic treatment of human cases can reduce the duration of fever but may not reduce mortality. Penicillin is the drug of choice, but alternatives are doxycycline, ampicillin, erythromycin, cephalosporin's and quinolone antibiotics [59].

Table 2: Antimicrobial agents recommended for treatment and chemoprophylaxis of leptospirosis, table from [56]

\begin{tabular}{lll}
\hline Indication & Compound & Dosage \\
\hline Chemoprophylaxis & Doxycycline & 200 mg orally once-weekly \\
Treatment of mild leptospirosis & Doxycycline & 100 mg twice-daily \\
& Ampicilline & $500 \mathrm{mg}$ every $6 \mathrm{~h}$ \\
Treatment of moderate to severe leptospirosis & Penicillin G & $1.5 \mathrm{MU}$ intravenously every 6 h \\
& Ceftriaxone & $1 \mathrm{~g}$ intravenously every 24 h \\
\hline
\end{tabular}

\section{CONCLUSION}

Clinical diagnosis of leptospirosis can be challenging because signs are nonspecific. Nonspecific clinical signs of leptospirosis including fever, inappetence, vomiting, abdominal pain, diarrhoea, polyuria/ polydipsia, myalgia, jaundice, epistaxis, and haematuria may suggest disease but are not diagnostic. Clinical, pathologic changes can be variable depending on the stage of infection. Diagnosis is most often initially based on clinical suspicion and confirmed later by laboratory methods. 
Rapid diagnostic tests should ideally be accurate, simple to use, relatively inexpensive, easy to interpret, stable under extreme conditions with little or no processing and give the results within 1-2 $\mathrm{h}$.

Currently, no diagnostic technique is completely satisfactory. Culture requires a one-month incubation and does not allow making a quick diagnosis. The fastidious organism requires a special medium for isolation. The technique is laborious, time-consuming and is not possible in small laboratories. Contamination of culture media by other micro-organisms or by saprophytic leptospirosis is common in routine practice and the successful isolation rate is less due to prior use of antibiotics and the acidity problems.

Silver stain is the traditional method for demonstration of spirochetes in tissue sections. However, difficulties arise when using silver stains because of extensive reticulin staining and inability to recognize leptospiral fragments especially if few organisms are present. Leptospires can be difficult to demonstrate in cases confirmed positive by other diagnostic methods and silver stains cannot identify altered leptospires, leptospire fragments or antigenic products.

Demonstration of leptospires by using dark-field microscopy appears to be a simple and rapid procedure, but it is not true in practice. Though the organism is present in the blood during the acute stage of the disease, the concentration is too low to allow detection by direct microscopy and the leptospiral shedding in urine is intermittent. A major disadvantage to dark-field microscopy testing is the inability to identify the infecting serovar. And the risk of false positives due to misinterpretation of fibrin or protein threads, cell debris and other artefacts can be high even for experts.

Polymerase chain reaction (PCR) on a blood sample is possible only for a few days during the first week of the disease. PCR-based diagnosis remains restricted to reference. It is unlikely that PCR will be widely applied in developing countries where the prompt diagnosis is most needed. Although the DNA from as few as 100 leptospires can be isolated, amplified and detected, it may not be possible differentiate the causative serovar.

MAT is complex and time-consuming, 14-21 strains have to be maintained in a culture which is often very difficult, reading results requires experienced personnel. It is not possible to distinguish between IgM and IgG antibodies. The interpretation of a single titre is not easy, so a second serum sample is required for demonstrating a raising titre which has a diagnostic significance and to confirm leptospirosis. Many laboratories and hospitals do not have the facilities required to perform the MAT. Furthermore, the MAT is influenced by vaccinal antibodies.

Although SAT and Elisa have high sensitivity at the acute phase more than MAT, they are not suitable for epidemiological studies, identification of strains and assessment of the probable infecting serogroup. As well as Elisa is Comparatively less specific.

Finally, there is no sensitive, specific, low-cost, rapid and widely available diagnostic test for leptospirosis. Thus, there is an urgent need to develop new techniques for an easy to use and quick detection of antibodies or antigens at the acute stage of the disease.

\section{CONFLICTS OF INTERESTS}

\section{Declared none}

\section{REFERENCES}

1. Levett PN. Leptospirosis. Clin Microbiol 2001;14:296-326.

2. Rao RS, Gupta N, Bhalla P, Agarwal SK. Leptospirosis in India and the rest of the World. Braz J Infect Dis 2003;7:178-93.

3. Dreyfus A, Wilson P, Benschop J, Verdugo C, Collins-Emerson J. Economic loss associated with leptospira seroprevalence. In: New Zealand Sheep, Cattle, and Deer. Institute of Veterinary Animal and Biomedical Sciences Massey University; 2013.

4. Fang F. Leptospirosis diagnostics and exposure at the human and animal interface in New Zealand. Massey University; 2014.

5. Toyokawa T, Makoto O, Nobuo K. Diagnosis of acute leptospirosis. Expert Rev Anti-Infect Ther 2011;9:111-21.
6. Manolito L, Marissa M, Rhona G, Raul P, Mario M, Cecilia S, et al. Leptospirosis. Philippine Society for Microbiology and Infectious Diseases; 2010. p. 1-66.

7. Tilahun Z, Reta D, Simenew K. Global epidemiological overview of leptospirosis. Int J Microbiol Res 2013;4:9-15.

8. Hartskeerl RA, Collares Pereira M, Ellis WA. Emergence, control and re-emerging leptospirosis: dynamics of infection in the changing world. Clin Microbiol Infect 2011;17:494-501.

9. Vijayachari P, Sharma S, Natarajaseenivasan K. Laboratory diagnosis. Leptospirosis, laboratory manual. Regional Medical Research Centre, Indian Council of Medical Research, Port Blair and World Health Organization; 2007. p. 27-45.

10. Burriel AR. Leptospirosis: an important zoonotic diseases. Current research technology and education topics in applied microbiology and microbial biotechnology. Badajoz: Formatex; 2010. p. 687-93.

11. WHO. Leptospirosis surveillance. Report 18 December; 2008.

12. Washington State Department of Health. Leptospirosis. Reporting and Surveillance Guidelines; 2015. p. 1-7.

13. Natarajaseenivasan K, Raja V, Narayanan R. Rapid diagnosis of leptospirosis in patients with different clinical manifestations by 16S rRNA gene-based nested PCR. Saudi J Biol Sci 2012;19:151-5.

14. Picardeau M, Bertherat E, Jancloes M, Skouloudis AN, Durski K, Hartskeerl RA. Rapid tests for diagnosis of leptospirosis: Current tools and emerging technologies. Diagn Microbiol Infect Dis 2014;78:1-8.

15. Picardeau M. Diagnosis and epidemiology of leptospirosis. Med Mal Infect 2013;43:1-9.

16. Faine S. Leptospira and leptospirosis. CRC Press Inc; 1994.

17. Connaissances document destine aux professionnel de sante. Le point sur la leptospirose France; 2012. p. 1-2.

18. Bharti A, Nally J, Ricaldi J, Matthias M, Diaz M, Lovett M, et al. Leptospirosis: a zoonotic disease of global importance. Lancet Infect Dis 2003;3:757-71.

19. Faine P, Adler S, Bolin B, Perolat C. Leptospira and Leptospirosis. 2ed ed Melbourne. Australia MediSci; 1999.

20. Andersson L. Leptospirosis the neglected disease Clinical picture and epidemiology at the Andaman Islands India. Göteborg University Sweden; 2012.

21. Musso D, La Scola B. Laboratory diagnosis of leptospirosis: a challenge. J Microbiol Immunol Infect 2013;46:245-52.

22. Draghi M, Brihuega B, Benitez D, Sala J, Biotti G, Pereyra M. Leptospirosis outbreak in calves from Corrientes Province, Argentina. Rev Argent Microbiol 2011;43:42-4.

23. WHO. Human leptospirosis: guidance for diagnosis, surveillance and control. WHO Libr 2003;45:1-109.

24. Ben Adler, Alejandro de la Peña Moctezuma. Leptospira and leptospirosis. Vet Microbiol 2010;140:287-96.

25. Plank R, Dean D. Overview of the epidemiology, microbiology, and pathogenesis of Leptospira spp. in humans. Microbes Infect 2000;2:1265-76.

26. The center for food security and public health. Leptospirosis. lowa State University; 2013. p. 1-10.

27. Boonsilp S, Thaipadungpanit J, Amornchai P, Wuthiekanun V, Chierakul W, Limmathurotsakul D, et al. Molecular detection and speciation of pathogenic Leptospira spp. in blood from patients with culture-negative leptospirosis. BMC Infect Dis 2011;11:338.

28. Finger M, Roque I, Filho D, Leutenegger C, Estrada M, Ullmann L. Serological and molecular survey of Leptospira spp. among cart horses from an endemic area of human leptospirosis in Curitiba, southern Brazil. Rev Inst Med Trop Sao Paulo 2014;56:473-6.

29. Magajevski F, Silva Girio R, Mathias L, Myashiro S, Genovez M, Scarcelli P. Detection of Leptospira spp. in the semen and urine of bulls serologically reactive to leptospira interrogans serovar Hardjo. Brazilian J Microbiol 2005;36:43-7.

30. Zakeri S, Sepahian N, Afsharpad M, Esfandiari B, Ziapour P, Djadid N. Molecular epidemiology of Leptospirosis in northern Iran by nested polymerase chain reaction/restriction fragment length polymorphism and sequencing methods. Am J Trop Med Hyg 2010;82:899-903.

31. Costa F, Hagan J, Calcagno J, Kane M, Torgerson P, Martinez-Silveira $\mathrm{M}$, et al. Global morbidity and mortality of leptospirosis: a systematic review. PLoS Neglected Trop Dis 2015;9:1-19. 
32. Natarajaseenivasan K, Vijayachari P, Sharma S, Sugunan A, Vedhagiri K, Selvin J, et al. FlaB PCR-based identification of pathogenic leptospiral isolates. J Microbiol Immunol Infect 2010;43:62-9.

33. de Abreu Fonseca C, Teixeira de Freitas V, Caló Romero E, Spinosa C, Arroyo Sanches M, Da Silva M, et al. Polymerase chain reaction in comparison with serological tests for early diagnosis of human leptospirosis. Trop Med Int Health 2006;11:1699-707.

34. Haake, Levett. Leptospirosis in humans. Curr Top Microbiol Immunol 2015;387:1-33.

35. Cerqueira G, Picardeau M, A century of leptospira strain typing. Infect Genet Evol 2009;9:760-8.

36. Rao R, Gupta N, Bhalla P, Agarwal S. Leptospirosis in India and the rest of the world. Braz J Infect Dis 2003;7:178-93.

37. Loureiro G, Martins S, Lilenbaum W. Laboratorial diagnosis of animal leptospirosis, Diagnóstico laboratorial de leptospirose animal. Revista Brasileira de Ciência Veterinária 2013;20:119-26.

38. Ohio Department of Health Disease Fact Sheet. Leptospira interrogations; 2005. p. 1-21.

39. Sritharan M. Insights into leptospirosis, a neglected disease. Department of Animal Sciences. India; 2003. p. 167-92.

40. Guidelines for the Prevention of Leptospirosis. Communicable Disease Control; 2008. p. 1-12.

41. Levett P, Haake D. Leptospira Species (Leptospirosis); 1998. p. 1-8.

42. Shivakumar S, Glasg F. Guidelines for diagnosis of leptospirosis-role of the mat. Govt Stanley Medical College and Hospital Chennai; 2008. p. 1-26.

43. Kumar S. Indian Guidelines for the Diagnosis and Management of Human Leptospirosis. API India 2012;24-9. Doi:10.13140/2.1.1759.9682.

44. Niloofa R, Fernando N, de Silva N, Karunanayake L, Wickramasinghe $\mathrm{H}$, Dikmadugoda $\mathrm{N}$, et al. Diagnosis of leptospirosis: comparison between microscopic agglutination test, IgM-ELISA and IgM rapid Immunochromatography test. PLoS One 2015;10:1-12.

45. Chen H, Zhang Z, Halsey E, Guevara C, Canal E, Hall E, et al. Detection of Leptospira-specific antibodies using a recombinant antigen-based enzyme-linked immunosorbent assay. Am J Trop Med Hyg 2013;89:1088-94.

46. Sabir M, Hatta M. Detection of IgM to leptospira agent with ELISA and leptodistick method. Biocelebes 2011;5:8-12.

47. Sakhaee E, Abdollahpour G, Bolourchi M, Tabatabayi A, Tabrizi $\mathrm{S}$. Serologic and bacteriologic diagnosis of bovine leptospirosis in Tehran suburb dairy farms. Iranian J Veterinary Res 2007;8:325-32.
48. Desakorn V, Wuthiekanun V, Thanachartwet V, Sahassananda D, Chierakul W, Apiwattanaporn A, et al. Accuracy of a commercial IgM ELISA for the diagnosis of human leptospirosis in Thailand. Am J Trop Med Hyg 2012;86:524-7.

49. Winslow W, Merry D, Pirc M. Evaluation of a commercial enzyme-linked immunosorbent assay for detection of immunoglobulin $\mathrm{M}$ antibody in the diagnosis of human leptospiral infection. J Clin Microbiol 1997;35:1938-42.

50. Chirathaworn C, Kaewopas Y, Poovorawan Y, Suwancharoen D. Comparison of a slide agglutination test, LeptoTek Dri-Dot, and IgM-ELISA with microscopic agglutination test for Leptospira antibody detection. Southeast Asian J Trop Med Public Health 2007;38:1111-4.

51. Lilenbaum W, Ristow P, Fráguas S, Da Silva E. Evaluation of a rapid slide agglutination test for the diagnosis of acute canine leptospirosis. Rev Latinoam Microbiol 2002;44:124-8.

52. Galton R, Powers M, Hall D, Cornell A. A rapid macroscopicslide screening test for the serodiagnosis of leptospirosis. Am J Vet Res 1958;72:505-12.

53. Stoenner E, Davis H. Further observations on leptospiral plate antigens. Am J Vet Res 1967;28:259-66.

54. Brandão A, Camargo E, da Silva E, Silva V, Abrão R. Macroscopic agglutination test for rapid diagnosis of human leptospirosis. J Clin Microbiol 1998;36:3138-42.

55. Pappas G, Cascio A. Optimal treatment of leptospirosis: queries and projections. Int J Antimicrob Agents 2006;28:491-6.

56. Edwards C, Levett P. Prevention and treatment of leptospirosis. Expert Rev Anti Infect Ther 2004;2:293-8.

57. Dutta T, Christopher M. Leptospirosis an overview. J Assoc Physicians India 2005;53:545-51.

58. Spichler A, Moock M, Chapola E, Vinetz J. Weil's disease: an unusually fulminant presentation characterized by pulmonary hemorrhage and shock. Brazilian J Infect Dis 2005;9:336-40.

59. Monika J, Nigam R, Reeni M. A review of laboratory techniques for detecting leptospirosis. Int J Curr Pharm Res 2015;7:1-8.

60. Gonsalez C, Casseb J, Monteiro F, Paula-Neto J, Fernandez R, Silva M, et al. Use of doxycycline for leptospirosis after highrisk exposure in São Paulo, Brazil. Rev Inst Med Trop Sao Paulo 1998;40:59-61.

61. Uma K, Mini J, Ganesh A, Kalpan S. In vitro antimicrobial activity of the siddha drugs seenthil sarkarai and nilavembu kudineer against leptospira. Int J Pharm Pharm Sci 2012;4:76-9.

\section{How to cite this article}

- Waleed AL-Orry, Moustapha Arahou, Rachida Hassikou, Zakaria Mennane. A review of laboratory diagnosis and treatment of leptospirosis. Int J Pharm Pharm Sci 2016;8(12):7-13. 